\title{
Differing commissioning arrangements may contribute to geographic variation in clinical management of digital ulcers in systemic sclerosis
}

\author{
Authors: Elizabeth Reilly, ${ }^{\mathrm{A}}$ Randa Alshakh, ${ }^{\mathrm{B}}$ Celia Beynon, ${ }^{\mathrm{C}}$ Matthew Cates, ${ }^{\mathrm{D}}$ Dhivya Das, ${ }^{\mathrm{E}}$ Shuja Majeed, ${ }^{\mathrm{F}}$ \\ Ahsan Memon, ${ }^{\mathrm{G}}$ Patrick O'Beirn, ${ }^{\mathrm{H}}$ James Ritchie ${ }^{\mathrm{I}}$ and John D Pauling ${ }^{\mathrm{J}}$
}

Phosphodiesterase inhibitors (such as sildenafil) and endothelin receptor antagonist, bosentan, are effective for digital ulcer disease in systemic sclerosis (SSc-DU) and are endorsed in international treatment recommendations. Commissioning of high-cost drugs, such as bosentan, however, differs across devolved nations of the UK. We report a multicentre service evaluation project to examine 'real world' management of SSc-DU before and following the 2015 UK Scleroderma Study Group (UKSSG) guidance, across southwest (SW) England and Wales. Results showed that iloprost and sildenafil use for SSc-DU was higher in patients in Wales prior to 2015. Between 2015-2017, sildenafil use for SSc-DU increased in SW England while remaining stable in Wales. Bosentan use for SSc-DU after 2015 in SW England increased, while remaining stable and proportionately lower in Wales. These findings demonstrate that differing commissioning guidance across devolved nations of the UK seems to contribute to geographic variation in patient care.

KEYWORDS: Systemic sclerosis, digital ulcer, endothelin receptor antagonist, phosphodiesterase inhibitors, scleroderma

DOI: $10.7861 /$ clinmed.2020-0031

\section{Introduction}

Systemic sclerosis (SSc) is a rare multisystem disease of unknown aetiology characterised by vasculopathy and aberrant tissue

Authors: ${ }^{\text {A }}$ linical research fellow, Royal National Hospital for Rheumatic Diseases, Bath, UK and University of Bath, Bath, UK; ${ }^{B}$ rheumatology registrar, Bristol Royal Infirmary, Bristol, UK; Crheumatology registrar, University Hospital of Wales, Cardiff, UK;

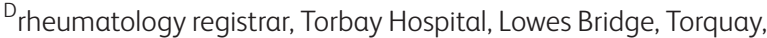

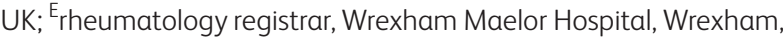
UK; ${ }^{F}$ rheumatology registrar, Great Western Hospital, Swindon, UK; ${ }^{G}$ rheumatology registrar, Royal Devon and Exeter Hospital, Exeter, UK; ${ }^{{ }^{H}}$ rheumatology registrar, University Hospital of Wales, Cardiff, UK; ${ }^{\mathrm{I}}$ rheumatology registrar, Weston General Hospital, Westonsuper-Mare, UK; ${ }^{3}$ consultant rheumatologist, Royal National Hospital for Rheumatic Diseases, Bath, UK and University of Bath, Bath, UK remodelling. Digital vasculopathy is an important feature of SSC and manifests clinically as symptoms of Raynaud's phenomenon (RP) and abnormal capillary morphology at the nailfold which are present in virtually all patients with SSc. ${ }^{1}$ Structural and functiona digital vasculopathy can result in significant tissue ischaemia and tissue damage. Digital ulcers (DU) occur in approximately half of patients with $\mathrm{SSc}^{2,3} \mathrm{SSc}$-DU are a major cause of disease-related morbidity in SSc. ${ }^{4,5}$ For some patients, DU occur as an isolated phenomenon but SSc-DU are recurrent in nature and relatively refractory to intervention in approximately $10 \%$ of patients.

The acute management for SSc-DU includes optimising analgesia and oral vasodilators (for example with calcium channel blockers, angiotensin II receptor antagonists, selective serotonin reuptake inhibitors and phosphodiesterase inhibitors) and/or intravenous vasodilator therapy (iloprost). Potential contributing factors, such as large vessel disease, should be treated aggressively and investigation and treatment of underlying infection is important. Magnetic resonance imaging can be useful to exclude deep infection and surgical debridement may be helpful in some circumstances.

The use of potent vasodilators, such as phosphodiesterase inhibitors (eg sildenafil), and the endothelin receptor antagonist bosentan are effective for secondary SSc-DU prevention and are endorsed in national and international treatment recommendations for the management of SSc. ${ }^{6-12}$

The existing commissioning of high-cost drugs, such as bosentan, differs across the devolved nations of the UK. The 2015 NHS England Clinical Commissioning Policy on the use of sildenafil and bosentan for the management of SSC-DU closely aligns with contemporary UK-based guidelines produced by the UK Scleroderma Study Group (UKSSG) and by the British Society of Rheumatology (BSR). ${ }^{8-10}$ Prior to 2015, reimbursement for treatment with bosentan and sildenafil for SSC-DU in England required individual funding requests (IFRs), whereas in Wales, clinicians could prescribe sildenafil without prior authorisation. In 2009, the All Wales Medicines Strategy Group published an appraisal notice indicating that bosentan was not endorsed for use within NHS Wales for the treatment of DU in severe Raynaud's disease, requiring clinicians to prepare an IFR.

\section{Methods}

We undertook a multicentre service evaluation project to examine 'real world' management of SSc-DU before and following the 
2015 UKSSG guidance across south-west (SW) England and Wales. This collaborative service evaluation project was conducted by rheumatology trainees within the South West Audit Network (SWAN); comprising rheumatology centres across SW England and Wales. Clinical audit office and information governance approval was obtained at each site. Anonymised data on patient demographics, SSc-DU burden and SSC-DU management before and after 2015 was retrospectively collected from the case-notes of adult patients with an existing clinical diagnosis of SSc made prior to 2015 (according to the 2013 American College of Rheumatology / European League Against Rheumatism classification criteria), using an MS Access form between September and December 2017. Patients with pulmonary arterial hypertension (PAH) were excluded from the analysis (and patients attending a dedicated regional SSC-PAH clinic in Bath were not included in the audit). The indication for the use of sildenafil or bosentan in each case was confirmed to be SSC-DU. The routine use of intravenous prostanoids for both $\mathrm{RP}$ and SSc-DU disease rendered establishing the main treatment indication (often both) challenging and data was consequently pooled. Results were analysed using IBM SPSS Statistics package version 24.

\section{Results}

The case-notes of 284 SSc patients were retrospectively assessed (68\% SW England; $32 \%$ Wales); 11 of whom were excluded owing to the presence of SSc-PAH. The majority had limited cutaneous SSC (83.1\%) with a mean disease duration of 10.4 years (standard deviation (SD) 8.2). One-hundred and thirteen patients (39.8\%) had a history of DU prior to 2015 (85 in SW England). DU burden was available for 110 patients (a solitary DU in 23, 2-4 previous DU in 55 and $\geq 5$ previous DU in 32 patients). A higher burden of DU was associated with longer disease duration (9.0 vs 13.5 years; $p=0.04$ ) and higher vasodilator medication usage $(p=0.09)$. Intravenous iloprost $(89.3 \%$ vs $48.2 \% ; p=0.0001)$ and sildenafil (32.1\% vs $11.8 \%$; $p=0.019)$ use for SSc-DU was higher in patients managed in Wales compared to England prior to 2015 (Table 1). Sixty-one patients experienced new DU between 2015-2017 (recurrence of DU in 46 patients and first DU in 15 patients). Between 2015-2017, sildenafil use for SSC-DU increased significantly in SW England ( $23.9 \%$ vs $11.8 \%$; $p=0.049$ ) while remaining stable in Wales. Bosentan use for SSc-DU in SW England increased by $47 \%$ after 2015 (5.9\% to $8.7 \%$; $p=0.57)$, while remaining stable and proportionately lower in Wales over this period $(8.7 \%$ vs $2.8 \%$; $p=0.44)$.

\section{Discussion}

The NHS England clinical commissioning policy enables specialist centres in England to prescribe sildenafil and/or bosentan for refractory SSc-DU disease in line with contemporary UKSSG and BSR guidance. ${ }^{9-11}$ The NHS England policy anticipated $\sim 1.7 \%$ of all SSc patients would satisfy the criteria for treatment with bosentan. Our regional data suggests $\sim 3 \%$ of all SSc patients have been managed with bosentan for SSc-DU. The high prevalence of recurrent SSc-DU ( $50 \%$ of those with a history of DU at 2015) and proportionately higher sildenafil use suggests sildenafil therapy may have prevented escalation to bosentan therapy in some patients in England. Sildenafil prescribing for SSc-DU in SW England rose to match that of Wales following the 2015 NHS England commissioning guidance. In contrast, while bosentan prescribing for SSc-DU rose in England after 2015, it remained proportionately lower and remained stable in Wales (where IFRs were still required to prescribe bosentan).

Our service evaluation project benefits from being multicentre in nature. However, not all units in the region took part and it is therefore expected that not all patients in the region with SSc could be captured. Furthermore, other factors may have influenced prescribing practices across sites, such as prior clinical experience of bosentan and sildenafil prescribing influencing differing clinician preferences in their management of SSC-DU. Nevertheless, our findings suggest high-cost drug use for rare autoimmune rheumatic diseases such as SSc may be influenced by differing commissioning arrangements within the devolved nations of the UK. Consequently, this may result in geographic variation in clinical practice and inequitable patient access to effective treatments for important complications such as SSC-DU. Our findings may be replicated across other diseases in which divergent commissioning guidance within the separate devolved nations may also be leading to inequitable access to high-cost drugs.

Table 1. Use of intravenous prostanoids, sildenafil and bosentan for management of digital ulcer disease in systemic sclerosis in south-west England and Wales prior to and after 2015

\begin{tabular}{|c|c|c|c|c|c|c|c|c|c|}
\hline \multirow{2}{*}{$\begin{array}{l}\text { SSc-DU } \\
\text { management } \\
\text { prior to 2015, } \\
\text { n (\%) }\end{array}$} & \multicolumn{3}{|c|}{ All regions, $n=113$} & \multicolumn{3}{|c|}{ South-west England, $n=85$} & \multicolumn{3}{|c|}{ Wales, $n=28$} \\
\hline & $66(58.4)$ & $19(16.8)$ & $6(5.3)$ & $\begin{array}{l}41(48.2) \\
p=0.0001 \\
\text { vs Wales }\end{array}$ & $\begin{array}{l}10(11.8) \\
p=0.019, \text { vs } \\
\text { Wales }\end{array}$ & $5(5.9)$ & $25(89.3)$ & $9(32.1)$ & $1(3.6)$ \\
\hline & \multicolumn{3}{|c|}{ All regions, $n=128$} & \multicolumn{3}{|c|}{ South-west England, $\mathrm{n}=92$} & \multicolumn{3}{|c|}{ Wales, $n=36$} \\
\hline $\begin{array}{l}\text { SSc-DU } \\
\text { management } \\
\text { at } 2017, \mathrm{n}(\%)\end{array}$ & $79(61.7)$ & $33(25.8)$ & $9(7.0)$ & $49(53.3)$ & $\begin{array}{l}22(23.9) \\
p=0.049, v s \\
\text { prior to } 2015\end{array}$ & $8(8.7)$ & $30(83.3)$ & $11(30.6)$ & $1(2.8)$ \\
\hline
\end{tabular}




\section{Key points}

> Intravenous iloprost and sildenafil use for SSc-DU was higher in patients managed in Wales compared to England prior to 2015.

> Between 2015-2017, sildenafil use for SSc-DU increased significantly in SW England while remaining stable in Wales.

$>$ Bosentan use for SSC-DU after 2015 in SW England increased by $47 \%$, while remaining stable and proportionately lower in Wales over this period.

$>$ Differing commissioning guidance across devolved nations of the UK seems to contribute to geographic variation in patient care.

\section{Conflicts of interest}

Dr Pauling reports research grants, personal fees and non-financial support from Actelion Pharmaceuticals. Dr Pauling also reports personal fees from Boehringer Ingelheim and from Sojournix Pharma outside of the submitted work. Dr Reilly undertook this service evaluation project during an out-of-programme experience (OOPE) supported, in part, with an unrestricted research grant from Actelion Pharmaceuticals.

\section{Funding}

This work was supported by an unrestricted research grant from Actelion Pharmaceuticals.

\section{References}

1 Walker U, Tyndall A, Czirják L et al. Geographical variation of disease manifestations in systemic sclerosis: a report from the EULAR Scleroderma Trials and Research (EUSTAR) group database. Ann Rheum Dis 2009;68:856-62.

2 Hughes M, Pauling JD. Exploring the patient experience of digital ulcers in systemic sclerosis. Semin Arthritis Rheum 2018;48:888-94.
3 Steen V, Denton CP, Pope JE, Matucci-Cerinic M. Digital ulcers: overt vascular disease in systemic sclerosis. Rheumatology (Oxford) 2009;48 (Suppl 3):iii19-24.

4 Hughes M, Pauling JD, Jones ] et al. A multi-centre qualitative study exploring the patient experience of digital ulcers in systemic sclerosis. Arthritis Care Res (Hoboken). 2019:[Epub ahead of print].

5 Nihtyanova SI, Brough GM, Black CM, Denton CP. Clinical burden of digital vasculopathy in limited and diffuse cutaneous systemic sclerosis. Ann Rheum Dis 2008;67:120-3.

6 Matucci-Cerinic M, Denton CP, Furst DE et al. Bosentan treatment of digital ulcers related to systemic sclerosis: results from the RAPIDS-2 randomised, double-blind, placebo-controlled trial. Ann Rheum Dis 2011;70:32-8.

7 Korn JH, Mayes M, Matucci Cerinic M et al. Digital ulcers in systemic sclerosis: prevention by treatment with bosentan, an oral endothelin receptor antagonist. Arthritis Rheum 2004;50:3985-93.

8 Tingey T, Shu J, Smuczek J, Pope J. Meta-analysis of healing and prevention of digital ulcers in systemic sclerosis. Arthritis Care Res (Hoboken) 2013;65:1460-71.

9 NHS England. Clinical commissioning policy: Sildenafil and bosentan for the treatment of digital ulceration in systemic sclerosis. NHS, 2015

10 Denton CP, Hughes M, Gak N et al. BSR and BHPR guideline for the treatment of systemic sclerosis. J Rheumatol 2016;55:1906-10.

11 Hughes M, Ong VH, Anderson ME et al. Consensus best practice pathway of the UK Scleroderma Study Group: digital vasculopathy in systemic sclerosis. Rheumatology (Oxford) 2015;54:2015-24.

12 Kowal-Bielecka O, Fransen ], Avouac ] et al. Update of EULAR recommendations for the treatment of systemic sclerosis. Ann Rheum Dis 2017;76:1327-39.

Address for correspondence: Dr Elizabeth Reilly, Department of Rheumatology, Royal National Hospital for Rheumatic Diseases, Royal United Hospitals Bath NHS Foundation Trust, Combe Park, Bath BA1 3NG, UK.

Email: elizabethreilly2@nhs.net 